\title{
A functional inequality for real-analytic functions
}

\section{Bert Jagers ANd Horst Alzer}

Summary. For $f \in C^{n}(\mathbb{R})$ and $0 \leqslant t \leqslant x$ let $J_{n}(f, t, x)=(-1)^{n} f(-x) f^{(n)}(t)+f(x) f^{(n)}(-t)$. We prove that the only real-analytic functions satisfying $J_{n}(f, t, x) \geqslant 0$ for all $n=0,1,2, \ldots$ are the exponential functions $f(x)=c e^{\lambda x}, c, \lambda \in \mathbb{R}$. Further we present a nontrivial class of real-analytic functions satisfying the inequalities $J_{0}(f, x, x) \geqslant 0$ and $\int_{0}^{x}(x-t)^{n-1} J_{n}(f, t, x) d t \geqslant 0(n \geqslant 1)$.

\section{Introduction}

There exist many interesting inequalities involving a real-analytic function and its Taylor polynomial. Two of the best known examples state that the cosine and sine functions are enveloped by their Maclaurin series expansion; that is,

$(-1)^{n+1}\left\{\cos (x)-\sum_{i=0}^{n}(-1)^{i} \frac{x^{2 i}}{(2 i) !}\right\}$ and $(-1)^{n+1}\left\{\sin (x)-\sum_{i=0}^{n}(-1)^{i} \frac{x^{2 i+1}}{(2 i+1) !}\right\}$

are both nonnegative for all $x \in \mathbb{R}$ and all $n \in \mathbb{N}=\{0,1,2, \ldots\}$. See e.g. [2, pp. 26-29] and [3]. Two related examples, which we could not localize in the literature, and which involve both the cosine and sine functions as well as their Taylor polynomials in a single inequality, are

$$
\left|\sin (x) \sum_{i=0}^{[n / 2]}(-1)^{i} \frac{x^{2 i}}{(2 i) !}-\cos (x) \sum_{i=0}^{[(n-1) / 2]}(-1)^{i} \frac{x^{2 i+1}}{(2 i+1) !}\right| \leqslant \frac{|x|^{n+1}}{(n+1) !}
$$

and

$$
\left|\cos (x) \sum_{i=0}^{[n / 2]}(-1)^{i} \frac{x^{2 i}}{(2 i) !}+\sin (x) \sum_{i=0}^{[(n-1) / 2]}(-1)^{i} \frac{x^{2 i+1}}{(2 i+1) !}-1\right| \leqslant \frac{|x|^{n+1}}{(n+1) !} .
$$

AMS (1991) subject classification: Primary 39B72, 26D10. Secondary 26E05.

Manuscript received June 12, 1991, and in final form, October 24, 1991. 
Inequalities (1.1) and (1.2) are immediate consequences of the identities

$$
\begin{aligned}
\frac{d^{n}}{d x^{n}} \frac{\sin (x)}{x} & =(-1)^{n} \frac{n !}{x^{n+1}}\left\{\sin (x) \sum_{i=0}^{[n / 2]}(-1)^{i} \frac{x^{2 i}}{(2 i) !}-\cos (x) \sum_{i=0}^{[(n-1) / 2]}(-1)^{i} \frac{x^{2 i+1}}{(2 i+1) !}\right\} \\
& =x^{-n-1} \int_{0}^{x} t^{n} \sin (t+(n+1) \pi / 2) d t
\end{aligned}
$$

and

$$
\begin{aligned}
\frac{d^{n}}{d x^{n}} \frac{1-\cos (x)}{x}=(-1)^{n+1} \frac{n !}{x^{n+1}}\left\{\cos (x) \sum_{i=0}^{[n / 2]}(-1)^{i} \frac{x^{2 i}}{(2 i) !}\right. \\
\left.\quad+\sin (x) \sum_{i=0}^{[(n-1) / 2]}(-1)^{i} \frac{x^{2 i+1}}{(2 i+1) !}-1\right\} \\
=x^{-n-1} \int_{0}^{x} t^{n} \sin (t+n \pi / 2) d t .
\end{aligned}
$$

The identities in (1.3) follow easily from Leibniz' rule; a proof for the identities in (1.4) (which are due to T. H. Gronwall) can be found in [4]; see also [1, pp. 243-244].

The motivation to write this paper was given by the discovery of a doubleinequality involving the exponential functions $e^{x}$ and $e^{-x}$ and their Taylor polynomials of even and odd degree:

$$
e^{-x} \sum_{i=0}^{2 n-1} \frac{x^{i}}{i !}+e^{x} \sum_{i=0}^{2 n-1} \frac{(-x)^{i}}{i !} \leqslant 2 \leqslant e^{-x} \sum_{i=0}^{2 n} \frac{x^{i}}{i !}+e^{x} \sum_{i=0}^{2 n} \frac{(-x)^{i}}{i !}
$$

for all $x \in \mathbb{R}$ and all $n \in \mathbb{N}=\{0,1,2, \ldots\}$. These inequalities state that the realanalytic function $f$ given by $f(x)=e^{x}$ satisfies the double-inequality

$$
\begin{aligned}
& f(-x) \sum_{i=0}^{2 n-1} \frac{f^{(i)}(0)}{i !} x^{i}+f(x) \sum_{i=0}^{2 n-1} \frac{f^{(i)}(0)}{i !}(-x)^{i} \leqslant 2 f(x) f(-x) \\
& \leqslant f(-x) \sum_{i=0}^{2 n} \frac{f^{(i)}(0)}{i !} x^{i}+f(x) \sum_{i=0}^{2 n} \frac{f^{(i)}(0)}{i !}(-x)^{i},
\end{aligned}
$$

for all $x \in \mathbb{R}$ and all $n \in \mathbb{N}$. (In Section 2 we show that $f(x)=c e^{\lambda x}, c, \lambda \in \mathbb{R}$, solves (1.6).)

Let $\mathscr{F}$ denote the class of all real-analytic functions on $\mathbb{R}$. It is natural to ask: Is it possible to find all $f \in \mathscr{F}$ which fulfill (1.6) for all $x \in \mathbb{R}$ and all $n \in \mathbb{N}$ ? It is tempting to conjecture that the only real-analytic functions satisfying doubleinequality (1.6) are given by $f(x)=c e^{\lambda x}, c, \lambda \in \mathbb{R}$. However this conjecture is false 
as we shall demonstrate in Section 3, where we present a large class of nontrivial solutions of (1.6). The problem to find all solutions of (1.6) remains open.

The main aim of this paper is to solve a closely related problem which will be settled in the next section.

\section{The main result}

We start with basic definitions and notations. For $f \in C^{n}(\mathbb{R}), n \in \mathbb{N}$ and $t, x \in \mathbb{R}$ we define

$$
J_{n}(f, t, x)=(-1)^{n} f(-x) f^{(n)}(t)+f(x) f^{(n)}(-t),
$$

and

$$
I_{0}(f, x)=J_{0}(f, x, x)=2 f(x) f(-x),
$$

and, for $n \geqslant 1$,

$$
I_{n}(f, x)=\int_{0}^{x}(x-t)^{n-1} J_{n}(f, t, x) d t /(n-1) ! .
$$

If we use Taylor's formula with the integral expression for the remainder, then simple manipulations reveal that (1.6) is equivalent to

$$
I_{2 n}(f, x) \geqslant 0 \text { and } I_{2 n+1}(f, x) \geqslant 0 .
$$

Since $I_{n}(f, x)=I_{n}(f,-x)$, we can consider $I_{n}(f, x)$ for $x \geqslant 0$ only. The following useful results can be easily verified:

(i) Let $f \in C^{n}(\mathbb{R}), x \geqslant 0$. If

$$
J_{n}(f, t, x) \geqslant 0
$$

for all $t \in[0, x]$, then

$$
I_{n}(f, x) \geqslant 0 \text {. }
$$

(ii) For $f \in C^{n}(\mathbb{R})$, let $\varphi(x)=c f(\lambda x), c, \lambda \in \mathbb{R}$ and $x \in \mathbb{R}$. If $0 \leqslant t \leqslant x$, then

$$
J_{n}(\varphi, t, x)=c^{2}|\lambda|^{n} J_{n}(f,|\lambda| t,|\lambda| x)
$$

and

$$
I_{n}(\varphi, x)=c^{2} I_{n}(f, \lambda x)
$$


Hence:

(iii) If $f$ satisfies inequality (2.2) for all $t, x$ with $0 \leqslant t \leqslant x$, then so does $\varphi$. Similarly, if $I_{n}(f, x) \geqslant 0$ for all $x$, then $I_{n}(\varphi, x) \geqslant 0$ for all $x$, too.

(iv) If $f \in C^{n}(\mathbb{R}), n \geqslant 1$, then $\frac{\partial}{\partial t} J_{n-1}(f, t, x)=-J_{n}(f, t, x)$

In particular, if $f$ satisfies (2.2), then

$$
J_{n-1}(f, 0, x) \geqslant J_{n-1}(f, t, x) \geqslant J_{n-1}(f, x, x), \quad 0 \leqslant t \leqslant x .
$$

If we set $f(x)=e^{x}$, then we obtain $J_{n}(f, t, x) \geqslant 0$ for all $t \in[0, x]$ and $n \in \mathbb{N}$. Hence we conclude from (i) and (iii) that $\varphi(x)=c e^{\lambda x}, c, \lambda \in \mathbb{R}$, is a solution of (2.3), or equivalently, of (1.6) for all $n \in \mathbb{N}$. (A result already announced in Section 1.)

THEOREM. Let $f \in \mathscr{F}$. Then $f$ satisfies inequality (2.2), that is,

$$
(-1)^{n} f(-x) f^{(n)}(t)+f(x) f^{(n)}(-t) \geqslant 0
$$

for all $n \in \mathbb{N}$ and all $t, x$ with $0 \leqslant t \leqslant x$ if and only if $f$ is an exponential function, that $i s, f(x)=c e^{\lambda x}, c, \lambda \in \mathbb{R}$.

Proof. If $f(x)=c e^{\lambda x}$ and $0 \leqslant t \leqslant x$, then we have

$$
J_{2 n}(f, t, x)=2 c^{2} \lambda^{2 n} \cosh (\lambda(x-t)) \geqslant 0
$$

and

$$
J_{2 n+1}(f, t, x)=2 c^{2} \lambda^{2 n+1} \sinh (\lambda(x-t)) \geqslant 0 .
$$

The converse is more difficult to establish. Let $f \in \mathscr{F}$ satisfy inequality (2.2) for all $n \in \mathbb{N}$ and all $t, x$ with $0 \leqslant t \leqslant x$. Then we obtain

$$
2 f(x) f(-x)=J_{0}(f, x, x) \geqslant 0 \text { and } \frac{d}{d x} f(x) f(-x)=-J_{1}(f, x, x) \leqslant 0,
$$

which implies that $x \mapsto f(x) f(-x)$ is nonnegative and nonincreasing. Thus, if $f\left(x_{0}\right) f\left(-x_{0}\right)=0$ for some $x_{0} \geqslant 0$, then $f(x) f(-x)=0$ for all $x \geqslant x_{0}$ and, since $f$ is real-analytic, we get $f \equiv 0$. Hence we may suppose that $f(x) f(-x)>0$ for all $x$, 
which implies either $f(x)>0$ or $f(x)<0$ for all $x$. The latter case reduces to the first one by considering $-f$ instead of $f$, so we may suppose that $f$ is positive.

Let $A$ denote the set of all limit points of $f(x) / f(-x)$, as $x \rightarrow \infty$. Then three different cases arise: (1) $0 \in A$, (2) $\infty \in A$, and (3) $A \subset\left[c_{1}, c_{2}\right]$ with $0<c_{1} \leqslant c_{2}<\infty$. Case 2 can be reduced to case 1 by considering $x \mapsto f(-x)$ instead of $f$. We start with case 1 . Let $\left(x_{k}\right)$ be a sequence with $x_{k} \rightarrow \infty$ and $f\left(x_{k}\right) / f\left(-x_{k}\right) \rightarrow 0$, as $k \rightarrow \infty$. If we divide the left-hand side of (2.2) (with $x=x_{k}$ ) by $f\left(-x_{k}\right)$ and let $k$ tend to $\infty$, then we obtain

$$
(-1)^{n} f^{(n)}(t) \geqslant 0 \quad \text { for all } t \geqslant 0 \text { and } n \in \mathbb{N} .
$$

This means that $f$ is completely monotonic on $[0, \infty)$. From Bernstein's theorem [ 5 , p. 160] we conclude that $f$ can be represented by a (convergent) Laplace-Stieltjes integral

$$
f(t)=\int_{0}^{\infty} e^{-\imath u} d F(u), \quad t \geqslant 0,
$$

where $F$ is a bounded and nondecreasing function on $[0, \infty)$. By the following lemma we obtain that (2.4) is valid for all $t \in \mathbb{R}$.

LEMMA 1. Let $r$ be the abscissa of convergence of the complex Laplace-Stieltjes integral $L(z)=\int_{0}^{\infty} e^{-z u} d G(u)$, where $G$ is a nondecreasing function on $[0, \infty)$. Further let $g \in \mathscr{F}$ satisfy $g(t)=L(t)$ for all $t>s$ with $r \leqslant s<\infty$. Then $r=-\infty$ and $g(t)=L(t)$ for all $t \in \mathbb{R}$ (and, consequently,

$$
g(t)=\sum_{n=0}^{\infty} \frac{g^{(n)}(0)}{n !} t^{n}
$$

with $g^{(n)}(0)=(-1)^{n} \int_{0}^{\infty} u^{n} d G(u)$ for all $\left.t \in \mathbb{R}\right)$.

Proof of Lemma 1. Since $g \in \mathscr{F}$ and $L$ is analytic on the right-half plane determined by $r$, we obtain from the identity theorem for analytic functions that $g(t)=L(t)$ for all $t \in \mathbb{R}$ with $t>r$. This implies

$$
g^{(n)}(t)=L^{(n)}(t)=(-1)^{n} \int_{0}^{\infty} e^{-t u} u^{n} d G(u) \quad \text { for all } t>r .
$$

Suppose, for a contradiction, that $r>-\infty$. Then, by monotone convergence we have

$$
g^{(n)}(r)=\lim _{t \downarrow r} g^{(n)}(t)=(-1)^{n} \lim _{t \downarrow r} \int_{0}^{\infty} e^{-t u} u^{n} d G(u)=(-1)^{n} \int_{0}^{\infty} e^{-r u} u^{n} d G(u) .
$$


Since $g \in \mathscr{F}$ we may expand $g(r-\delta)$ in a Taylor series around $r$, where $\delta>0$ is sufficiently small. By Lebesgue's monotone convergence theorem we obtain for such $\delta$

$$
\begin{aligned}
g(r-\delta) & =\sum_{n=0}^{\infty}(-\delta)^{n} \frac{g^{(n)}(r)}{n !}=\sum_{n=0}^{\infty} \frac{\delta^{n}}{n !} \int_{0}^{\infty} e^{-r u} u^{n} d G(u) \\
& =\int_{0}^{\infty} e^{-r u}\left(\sum_{n=0}^{\infty} \frac{(\delta u)^{n}}{n !}\right) d G(u)=\int_{0}^{\infty} e^{-(r-\delta) u} d G(u) .
\end{aligned}
$$

The convergence of the last integral contradicts the fact that $r-\delta$ lies to the left of the abscissa of convergence $r$. It follows that $r=-\infty$, that $L$ is an entire function, and that $g$ is the restriction of $L$ to $\mathbb{R}$.

We continue with the analysis of case 1 . Let $n$ be odd, say $n=2 m+1$. Then we conclude from (2.2) with $t=x$ :

$$
0 \geqslant-J_{n}(f, x, x)=f(-x) f^{(2 m+1)}(x)-f(x) f^{(2 m+1)}(-x) .
$$

On the other hand, if we substitute $f(x)=\int_{0}^{\infty} e^{-x u} d F(u), x \in \mathbb{R}$, into the right-hand side of (2.5), then we get the reversed inequality, as is shown in a slightly more general setting by the following lemma.

LEMMA 2. Let $r$ be the abscissa of convergence of $g(z)=\int_{0}^{\infty} e^{-z u} d G(u)$, where $G$ is a nondecreasing function on $[0, \infty)$. Further let $n=2 m+1, r<0$, and $0<x<|r|$. Then $-J_{n}(g, x, x) \geqslant 0$, with equality holding if and only if $g(x)=c e^{-\lambda x}$ for all $x$, with $c, \lambda \geqslant 0$ (in which case $d G$ represents a point measure, concentrated in $\lambda$ ).

Proof of Lemma 2. We substitute $g(y)=\int_{0}^{\infty} e^{-y u} d G(u)$, with $y= \pm x(>r)$, into $-J_{n}(g, x, x)$. From Fubini's theorem and

$$
g^{(n)}(y)=(-1)^{n} \int_{0}^{\infty} e^{-y u} u^{n} d G(u)
$$

we obtain

$$
\begin{aligned}
-J_{n}(g, x, x)= & -\int_{0}^{\infty} e^{x u} d G(u) \int_{0}^{\infty} e^{-x v} v^{2 m+1} d G(v) \\
& +\int_{0}^{\infty} e^{-x u} d G(u) \int_{0}^{\infty} e^{x v} v^{2 m+1} d G(v) \\
= & 2 \int_{0}^{\infty} \int_{0}^{\infty} \sinh (x(v-u)) v^{2 m+1} d G(u) d G(v) \\
= & \int_{0}^{\infty} \int_{0}^{\infty} \sinh (x(v-u))\left(v^{2 m+1}-u^{2 m+1}\right) d G(u) d G(v) .
\end{aligned}
$$


Since the last integrand is positive for $u \neq v$, we obtain $-J_{n}(g, x, x) \geqslant 0$ with equality holding if and only if the set of all $(u, v)$ with $u \neq v$ is negligible with respect to the product measure.

From Lemma 2 and inequality (2.5) we conclude that (in case 1) $f(x)=c e^{-\lambda x}$ for all $x \in \mathbb{R}$, with $c, \lambda \geqslant 0$.

We now turn to case 3 . Our aim is to prove that in this case $f$ is a constant. Let $a \in A$, and let $\left(x_{n}\right)$ be a sequence with $x_{n} \rightarrow \infty$ and $f\left(x_{n}\right) / f\left(-x_{n}\right) \rightarrow a$, as $n \rightarrow \infty$. Then, by the same arguments as for the first case, it follows that the function $h: \mathbb{R} \rightarrow \mathbb{R}$ defined by $h(t)=f(t)+a f(-t)$ is completely monotonic on $[0, \infty)$. Since $f \in \mathscr{F}$ we obtain $h \in \mathscr{F}$. Thus, by the same reasoning as before, there exists a bounded and nondecreasing function $H$ on $[0, \infty)$, such that

$$
h(t)=f(t)+a f(-t)=\int_{0}^{\infty} e^{-t u} d H(u)
$$

for all $t \in \mathbb{R}$. Hence

$$
h(-t)=f(-t)+a f(t)=\int_{0}^{\infty} e^{t u} d H(u), \quad t \in \mathbb{R} .
$$

We show that $h$ is a constant. Indeed, suppose that $h$ is not constant. Then $t \mapsto h(-t)$ tends to $\infty$, as $t \rightarrow \infty$, whereas $t \mapsto h(t)$ is bounded on $[0, \infty)$. We have

$$
\frac{h\left(x_{n}\right)+h\left(-x_{n}\right)}{f\left(-x_{n}\right)}=(1+a)\left[1+\frac{f\left(x_{n}\right)}{f\left(-x_{n}\right)}\right] \text {, }
$$

and, since the sequence on the right-hand side converges as $n \rightarrow \infty$, we conclude that $f\left(-x_{n}\right) \rightarrow \infty$ as $n \rightarrow \infty$. Because $\left(h\left(x_{n}\right)\right)$ is bounded, we conclude from

$$
h\left(x_{n}\right)=f\left(x_{n}\right)+a f\left(-x_{n}\right)
$$

that $a=0$, which contradicts the assumption $a>0$. Therefore, $h$ is a constant, say $h=c$. Then we get $\left(1-a^{2}\right) f(t)=(1-a) c$, which implies either $f=c /(1+a)$ or $a=1$. In the latter case we get $A=\{1\}$. From $h(t)=f(t)+f(-t)=c$ and $f(t) / f(-t) \rightarrow 1$, as $t \rightarrow \infty$, we conclude $f(t) \rightarrow c / 2$ and $f(-t) \rightarrow c / 2$, as $t \rightarrow \infty$. This implies that the function $t \mapsto f(t) f(-t)$ attains the value $c^{2} / 4$ for both $t=0$ and $t \rightarrow \infty$. Since $t \mapsto f(t) f(-t)$ is nonincreasing, this function must be constant. Finally, from $f(t) f(-t)=c^{2} / 4$ and $f(t)+f(-t)=c$ we obtain $f=c / 2$.

This completes the proof of the theorem. 
We end this section with two remarks. The first states that the conclusion drawn from (2.5) and (2.6) holds under much weaker assumptions. The second shows that the theorem does not hold if real-analyticity is replaced by infinite differentiability.

REMARK 1 . The only positive three times differentiable functions $f$, which satisfy

$$
f(-x) f^{(2 m+1)}(x)-f(x) f^{(2 m+1)}(-x)=0
$$

for $m=0,1$ and all $x \in \mathbb{R}$, are given by $f(x)=c e^{\lambda x}$ with $c>0$.

Proof. By (2.7) with $m=0$ we obtain

$$
[\log (f(x) f(-x))]^{\prime}=\frac{f^{\prime}(x)}{f(x)}-\frac{f^{\prime}(-x)}{f(-x)}=0
$$

and so

$$
f(x) f(-x)=c_{1}
$$

for some constant $c_{1}>0$. Thus, from Leibniz' rule and (2.7) with $m=1$ we conclude

$$
0=(f(x) f(-x))^{\prime \prime \prime}=3 f^{\prime}(x) f^{\prime \prime}(-x)-3 f^{\prime \prime}(x) f^{\prime}(-x),
$$

or

$$
\left(f^{\prime}(x) f^{\prime}(-x)\right)^{\prime}=0
$$

This implies

$$
f^{\prime}(x) f^{\prime}(-x)=c_{2}
$$

for some constant $c_{2}$. From (2.8), (2.9) and (2.10) it follows that the function $\left(f^{\prime} \mid f\right)^{2}$ is constant. In fact,

$$
\left(\frac{f^{\prime}(x)}{f(x)}\right)^{2}=\frac{f^{\prime}(x) f^{\prime}(-x)}{f(x) f(-x)}=\frac{c_{2}}{c_{1}}=\lambda^{2}, \quad \text { say }
$$

which leads to $f(x)=c e^{\lambda x}$ or $f(x)=c e^{-\lambda x}$ with $c>0$. 
REMARK 2. Let $f(x)=0$ for $x \geqslant 0$ and $f(x)=e^{1 / x}$ for $x<0$. Then $f$ is an example of a $C^{\infty}$-function with $f^{(n)}(0)=0$ for all $n \in \mathbb{N}$, which does not belong to $\mathscr{F}$, but trivially satisfies inequality (2.2) for all $n \in \mathbb{N}$ and all $t, x$ with $0 \leqslant t \leqslant x$.

\section{A large class of real-analytic solutions of (2.3)}

In the last part of this paper we return to inequality (2.3), or equivalently to (1.6), and we provide a class of nontrivial functions satisfying (2.3). In particular we show that the set of real-analytic solutions of (2.3) contains not only $f(x)=c e^{\lambda x}$.

Proposition. Let $0<\alpha<\beta=2 \alpha$ and let $F$ be a nondecreasing function on $[\alpha, \beta]$. Then, $f: \mathbb{R} \rightarrow \mathbb{R}$ defined by

$$
f(y)=\int_{\alpha}^{\beta} e^{-y u} d F(u)
$$

belongs to $\mathscr{F}$ and satisfies (2.3) for all $n \in \mathbb{N}$ and all $x \geqslant 0$.

Proof. Since $[\alpha, \beta]$ is a finite interval, the abscissa of convergence for the Laplace transform (3.1) is equal to $-\infty$. Hence $f \in \mathscr{F}$ and $f^{(n)}(y)=(-1)^{n} \int_{\alpha}^{\beta} e^{-y u} u^{n} d F(u)$ for all $y \in \mathbb{R}$, and in particular we have $f^{(2 m)}(y) \geqslant 0$ for all $y \in \mathbb{R}$. This leads to $J_{2 m}(f, t, x) \geqslant 0$ for all $t$ with $0 \leqslant t \leqslant x$, and from the implication (i) in Section 2 we conclude $I_{2 m}(f, x) \geqslant 0$. It remains to show that $I_{2 m+1}(f, x) \geqslant 0$ for $x \geqslant 0$. By Fubini's theorem we have

$$
\begin{aligned}
(2 m) ! I_{2 m+1}(f, x)= & \int_{0}^{x}(x-t)^{2 m} \int_{\alpha}^{\beta} \int_{\alpha}^{\beta}\left(e^{x u-t v}-e^{-x u+t v}\right) v^{2 m+1} d F(u) d F(v) d t \\
= & \int_{\alpha}^{\beta} \int_{\alpha}^{\beta} \int_{0}^{x}(x-t)^{2 m}\left\{\frac{e^{x u-t v}-e^{-x u+t v}}{2} v^{2 m+1}\right. \\
& \left.+\frac{e^{x v-t u}-e^{-x v+t u}}{2} u^{2 m+1}\right\} d t d F(u) d F(v) \\
= & \int_{\alpha}^{\beta} \int_{\alpha}^{\beta} \int_{0}^{x}(x-t)^{2 m}\left\{\sinh (x u-t v) v^{2 m+1}\right. \\
& \left.+\sinh (x v-t u) u^{2 m+1}\right\} d t d F(u) d F(v) .
\end{aligned}
$$


We show that the inner integral is nonnegative. Because of symmetry we may assume $u \leqslant v$. If we set $v=\mu u$ with $1 \leqslant \mu \leqslant 2=\beta / \alpha$, then we have to verify:

$$
\mu^{2 m+1} \int_{0}^{x}(x-t)^{2 m} \sinh (u(x-t \mu)) d t+\int_{0}^{x}(x-t)^{2 m} \sinh (u(x \mu-t)) d t \geqslant 0 .
$$

Since $\sinh (u(x \mu-t)) \geqslant \sinh (u t(\mu-1)) \geqslant 0$ for $0 \leqslant t \leqslant x$, the second integral of (3.2) is nonnegative, and so it is enough to show:

$$
P(x, \mu)=\int_{0}^{x}(x-t)^{2 m} \sinh (u(x-t \mu)) d t \geqslant 0 .
$$

The function $P(x, \mu)$ is decreasing in $\mu$, so that it suffices to prove inequality (3.3) for the special case $\mu=2$. To establish this we set $\tau=t-x / 2$. Then we obtain

$$
\begin{aligned}
P(x, 2) & =\int_{-x / 2}^{0}\left(\frac{x}{2}-\tau\right)^{2 m} \sinh (-2 u \tau) d \tau+\int_{0}^{x / 2}\left(\frac{x}{2}-\tau\right)^{2 m} \sinh (-2 u \tau) d \tau \\
& \geqslant\left(\frac{x}{2}\right)^{2 m} \int_{-x / 2}^{0} \sinh (-2 u \tau) d \tau+\left(\frac{x}{2}\right)^{2 m} \int_{0}^{x / 2} \sinh (-2 u \tau) d \tau \\
& =\left(\frac{x}{2}\right)^{2 m} \int_{-x / 2}^{x / 2} \sinh (-2 u \tau) d \tau \\
& =0 .
\end{aligned}
$$

The following remark presents another set of nontrivial solutions of (2.3). We show that under certain conditions the sum of two exponential functions fulfills inequality (2.3).

REMARK 3. Let $0<\alpha<\beta$ and $0<p \leqslant q$. Then

$$
f(x)=p e^{-\alpha x}+q e^{-\beta x}
$$

satisfies (2.3) for all $n \in \mathbb{N}$ and all $x \geqslant 0$.

Proof. Because of implication (iii) given in Section 2, we may assume that $\alpha=1$ and $p+q=1$. As before, it suffices to consider the case of odd $n$. Let $n=2 m+1$; a simple calculation yields

$$
(2 m) ! I_{2 m+1}(f, x)>2 q \beta^{2 m} \int_{0}^{x}(x-t)^{2 m} g(t, x) d t
$$


with

$$
g(t, x)=p \beta \sinh (x-\beta t)+q \beta \sinh (\beta(x-t)) .
$$

Since $t \mapsto g(t, x)$ is strictly decreasing on $(0, x)$, there exists a number $t_{0} \in(0, x)$ such that

$$
g(t, x)>0 \quad \text { for } 0<t<t_{0} \text { and } g(t, x)<0 \quad \text { for } t_{0}<t<x \text {. }
$$

This implies

$$
\begin{aligned}
\int_{0}^{x}(x-t)^{2 m} g(t, x) d t & \geqslant\left(x-t_{0}\right)^{2 m} \int_{0}^{x} g(t, x) d t \\
& =\left(x-t_{0}\right)^{2 m}[q \cosh (\beta x)-p \cosh (x(\beta-1))-q+p \cosh (x)] \\
& \geqslant\left(x-t_{0}\right)^{2 m}(q-p)[\cosh ((\beta-1) x)-1] \\
& \geqslant 0 .
\end{aligned}
$$

It remains an open problem to determine all real-analytic functions $f$ satisfying (2.3) for all $n \in \mathbb{N}$ and for all $x \geqslant 0$. It seems useful to attack the problem at first under additional assumptions concerning $f$. Likely it is possible to find all solutions for certain subclasses of $\mathscr{F}$. In particular it would be interesting to determine all $f \in \mathscr{F}$ which are completely monotonic on $[0, \infty)$.

\section{REFERENCES}

[1] Mitrinović, D. S., Analytic inequalities. Springer, New York, 1970.

[2] Pólya, G. and Szegö, G., Aufgaben und Lehrsätze aus der Analysis, I. Springer, Berlin, 1970.

[3] Ross, D. K. and MAHAJAN, A., On enveloping series for some of the special functions, and on integral inequalities involving them. In: General inequalities 2 (E. F. Beckenbach, ed.). Birkhäuser, Basel, 1980, pp. $161-175$.

[4] Solution of Problem 331. Amer. Math. Monthly 20 (1913), 138.

[5] Widder, D. V., The Laplace transform. Princeton Univ. Press, Princeton, 1946.

Department of Mathematics, University of Twente, P.O. Box 217 , 7500 AE Enschede, The Netherlands.

Morsbacher Str. 10, D-S220 Waldbröl, Germany. 